This item was submitted to Loughborough's Research Repository by the author.

Items in Figshare are protected by copyright, with all rights reserved, unless otherwise indicated.

\title{
Parametric study of reduced span side tapering on a simplified model with wheels
}

PLEASE CITE THE PUBLISHED VERSION

https://doi.org/10.4271/2020-01-0680

PUBLISHER

SAE International

VERSION

AM (Accepted Manuscript)

\section{PUBLISHER STATEMENT}

This paper was accepted for publication in SAE Technical Papers and the definitive published version is available at https://doi.org/10.4271/2020-01-0680.

\section{LICENCE}

CC BY-NC 4.0

\section{REPOSITORY RECORD}

Varney, Max, Martin Passmore, Ryan Swakeen, and Adrian Gaylard. 2020. "Parametric Study of Reduced Span Side Tapering on a Simplified Model with Wheels". Loughborough University.

https://hdl.handle.net/2134/12162105.v1. 


\title{
Parametric Study of Reduced Span Side Tapering on a Simplified Model with Wheels
}

\author{
Author, co-author (Do NOT enter this information. It will be pulled from participant tab in \\ MyTechZone) \\ Affiliation (Do NOT enter this information. It will be pulled from participant tab in MyTechZone)
}

\begin{abstract}
Many modern vehicles have blunt rear end geometries for design aesthetics and practicality; however, such vehicles are potentially high drag. The application of tapering; typically applied to an entire edge of the base of the geometry is widely reported as a means of reducing drag, but in many cases, this is not practical on real vehicles. In this study side tapers are applied to only part of the side edge of a simplified automotive geometry, to show the effects of practical implementations of tapers.
\end{abstract}

The paper reports on a parametric study undertaken in Loughborough University's Large Wind Tunnel with the $1 / 4$ scale Windsor model equipped with wheels. The aerodynamic effect of implementing partial side edge tapers is assessed from a full height taper to a $25 \%$ taper in both an upper and lower body configuration. These were investigated using force and moment coefficients, pressure measurements and planar particle image velocimetry (PIV). These geometries showed that the drag reductions are maximised with a $50 \%$ span, generating a vertically symmetric wake and less taper drag contribution when compared to a full span taper.

\section{Introduction}

Aerodynamic drag is of concern to vehicle manufacturers because of its impact on electric vehicle range and emissions of $\mathrm{CO}_{2}$, and to consumers for its impact on fuel economy. Hence, finding new methods for reducing drag that can be implemented on real vehicles is becoming more important. This is a particularly pressing problem for SUVs because they make up 37.5\% of vehicles sold in Europe [1] and typically have large frontal areas and blunt rear end geometries that can result in high drag. Indeed for such vehicle up to $30 \%$ of the aerodynamic drag can be attributed to the rear surfaces $[2,3]$. The high rear end drag is a consequence of large scale flow separations from the trailing perimeter of the vehicle that generate shear layer bounded recirculating flows, that lower the pressure on the base of the vehicle.

Both passive and active methods have been applied to reducing rear end drag and both typically attempt to affect the recirculating region by manipulating the shear layers and increasing the base pressure and thereby reduce drag. Typical passive methods include side tapering [4-6], roof tapering [5-10] and cavities [11-13]. Whilst these methods can be effective, their implementation on a full scale vehicle may not fit the brand image or reduce rear capacity, (tapering) or have a significant impact on crash safety (cavities). Whilst active methods, such as base bleed [12], rotating surfaces [14] or edge blowing [15] would overcome this, their implementation is complex, potentially expensive and the reductions seen in some simplified studies have not yet been shown to scale up to real geometries.

Whether passive or active, all such methods alter the interaction between the shear layers around separation to better balance the wake and reduce the drag contribution of the base region of the vehicle. However, such modifications also typically exhibit an optimum condition whereby the reduction in drag contribution of the base is not offset by the newly introduced drag contributions, such as slant drag $[4-6,9]$ or device drag $[7,12]$.

Pavia et al. [4] and Perry et al. [5] both consider high aspect ratio side edge tapers (aspect ratio of 6.42) on a Windsor geometry and show the optimum at $0^{\circ}$ yaw to be a half angle of $12^{\circ}$ for both overall drag and the reduction in base pressure drag. Taper angles larger than this showed a rise in the pressure drag contribution from the tapered surface with a reduction in the drag on the base of the geometry or, if the taper is stalled, a return to a base pressure drag contribution similar to the $0^{\circ}$ taper but a larger overall drag due to the suction over the tapered surfaces.

Howell et al. [16] applied tapering to the upper $56 \%$ of a Windsor geometry on both the roof and the side edges. Justifying the partial tapers as being more realistic for real vehicles, as these cannot normally accommodate a full height body side trailing edge taper because of the requirement to cover the rear wheels. Howell reported a complex relationship between taper length and angle and the interaction between the roof taper and upper body side tapers. The upper bodyside tapers where also shown to supress the trailing vortex structures associated with a roof taper but introduced a vortex in the junction between the upper body taper and the un-tapered lower body (the shoulder) that generated downwash in the near wake.

Varney et al. [6] applied side edge tapering to a Generic SUV geometry at a small aspect ratio $(\sim 1.5)$, and for the same reasons as Howell applied them only to the upper $40 \%$ of the geometry, also producing the same shoulder junction as Howell et al. [16]. This produced an overall drag optimum at a half angle of $15^{\circ}$ but the study did not report the base pressure drag separately. It does however show some base pressure distributions, and, that the introduction of the $15^{\circ}$ taper changed the wake from being up-wash dominated to a downwash dominated wake, suggesting that despite being a relatively modest modification it has had a substantial effect on the wake structure that would be worthy of deeper investigation.

Varney et al. [17] also repeated the same geometries as Pavia et al. [4] and Perry et al. [5] on the Windsor geometry with wheels

Page 1 of 7 
showing a shift in the optimum to a broad $12^{\circ}$ to $20^{\circ}$ half taper angle. Additionally, they tested tapers only on the upper $50 \%$ of the Windsor body equipped with wheels, showing a greater total drag reduction than the full height tapering. This was attributed to longitudinal vortices re-symmetrising the wake, consistent with the literature that reports a more symmetric wake as resulting in lower drag [18-20], but no pressure or flow field measurements were presented to confirm this.

The work reported in this paper provides a more comprehensive study of the work from Varney et al. [17] to show how the reduced span tapering impacts the wake flow on the $1 / 4$ scale Windsor geometry with wheels, and in addition covers smaller increments of reduced span tapers. For completeness the experiment also explores the effect of applying tapering only to the lower part of a body, while this is perhaps unrealistic for real vehicles it provides a more complete understanding of the flow physics.

\section{Experimental Set Up}

\section{The Facilities}

All the testing has been carried out in the Loughborough University Large Wind Tunnel (Figure 1). The tunnel is an open return, closed working section design with a cross sectional area of $2.5 \mathrm{~m}^{2}$, capable of flow speeds up to $45 \mathrm{~m} / \mathrm{s}$ with a turbulence intensity of $0.2 \%$, a flow uniformity of $\pm 0.4 \%$ [21].

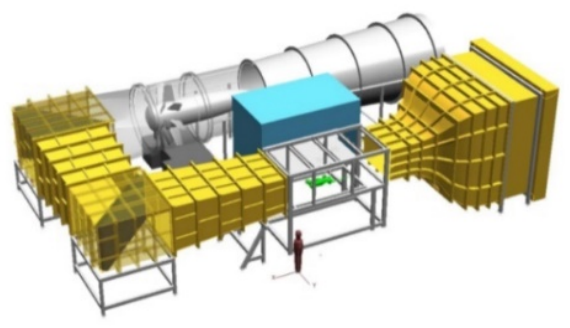

Figure 1. The Loughborough University wind tunnel [21].

\section{The Model}

The model used here is a variant on the $1 / 4$ scale Windsor geometry, modified to accept removable wheels (Figure 2 and Figure 3 ). The use of a simplified model Is a widely accepted approach for reducing sensitivity to Reynolds number while generating results that can be appropriately generalised. The addition of the wheels compared to other applications of this model results in a bulk wake flow that is comparable to a full scale vehicle. The Windsor model was selected over the simpler (Ahmed) model because it has height/width/length ratios that are more representative of all modern vehicles.

In this form the Windsor geometry has a blockage ratio of $4.7 \%$. It was tested at a non-dimensional ground clearance of 0.17 (defined as the ground clearance divided by the model body height). All forward facing radii are $50 \mathrm{~mm}$ to prevent separation around the front of the model; all other longitudinal and lateral edges are sharp. The geometries tested are made of either dimensionally stable model board or $3 \mathrm{D}$ printed from a rigid opaque material.

The principal dimensions of this variant of the Windsor geometry are: $1040 \mathrm{~mm}$ (length), $289 \mathrm{~mm}$ (height), $389 \mathrm{~mm}$ (width); with a $334 \mathrm{~mm}$ track, a $637.5 \mathrm{~mm}$ wheelbase and $150 \mathrm{~mm}$ diameter wheels.
The coordinate system defined by SAE J1594 [22] is used throughout this work, with the origin located at mid-wheelbase, mid-track and on the ground. The model is supported by four, $8 \mathrm{~mm}$ diameter pins protruding into the working section, on which the model is fastened. These are placed on either side of the model just behind the front wheels and just in front of the rear wheels to minimise the impact on the general flow, no drag tare for the pin protrusion has been included. The wheels have $\sim 4 \mathrm{~mm}$ ground clearance due to a recessed floor panel that maintains a non-dimensional ride height of 0.17 , illustrated in Figure 4.

The side edge tapers used here have a length along the slant of $45 \mathrm{~mm}$ ( $15.5 \%$ of the base height) resulting in a full body aspect ratio of 6.4 , with half angles of $0^{\circ}$ to $20^{\circ}$ in steps of $4^{\circ}$ (Figure 5, referred to as taper angle from here on in) with a sharp leading edge. These are applied symmetrically to the geometry, but also have the aspect ratio varied by removing some of the tapering. The range of spans considered is $25 \%, 50 \%$ and $75 \%$ on both the upper and lower body, illustrated in Figure 6.

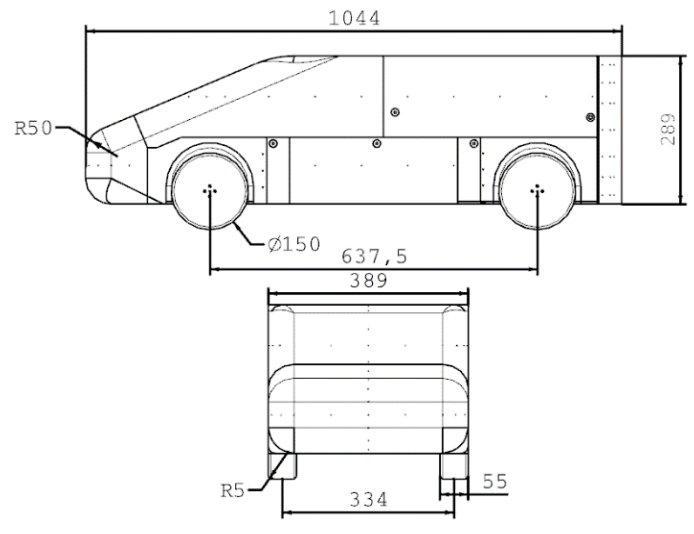

Figure 2. Windsor geometry with wheels.

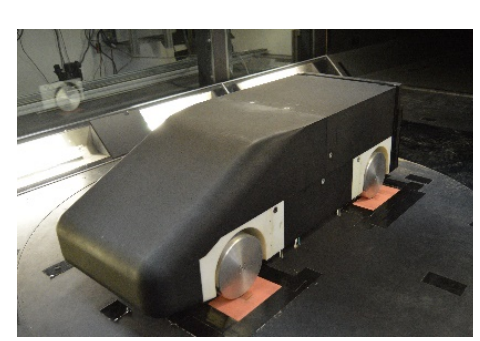

Figure 3. Windsor geometry with wheels installed in the Loughborough University wind tunnel.

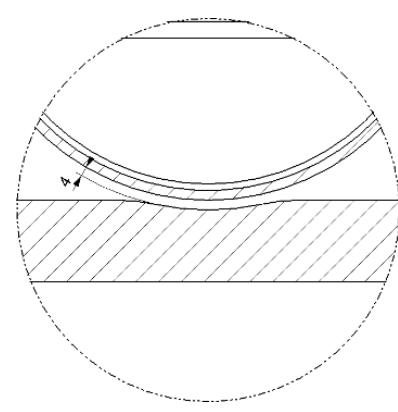

Figure 4. Illustration of the wheel ground clearance.

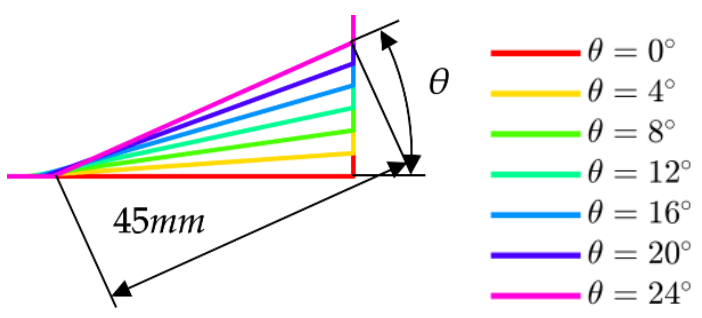

Figure 5. Geometric representation of taper values. 


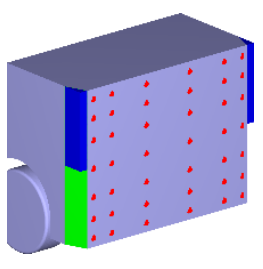

$50 \%$ lower body

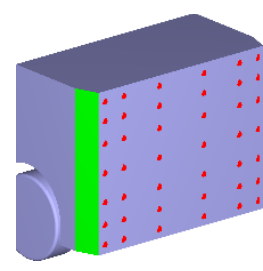

$100 \%$ Full body



$50 \%$ upper body
Figure 6. Explanation of reduced span tapering, including the pressure tapping distributions.

\section{Force Measurements}

All measurements were taken with an onset flow velocity of $40 \mathrm{~m} / \mathrm{s}$ (giving a length-based Reynolds number of $2.7 \times 10^{6}$ ). Balance data were sampled at $300 \mathrm{~Hz}$ for 300 seconds with an arithmetic mean taken during post processing.

All coefficient calculations use a corrected value for the velocity estimated using a continuity correction seen in Equation 2 [23]. The force coefficient calculation is presented in Equation 3.

$$
v_{\text {corrected }}=\frac{v \cdot A_{t}}{A_{t}-A_{m}}
$$

$\mathrm{A}_{\mathrm{t}}-$ Tunnel Area $\left(\mathrm{m}^{2}\right), \mathrm{A}_{\mathrm{m}}-$ Model Area $\left(\mathrm{m}^{2}\right), \mathrm{v}-\operatorname{Velocity}(\mathrm{m} / \mathrm{s})$

$$
\begin{aligned}
C_{\text {force }} & =\frac{\text { Force }}{\frac{1}{2} \rho A_{m} v_{\text {corrected }}^{2}} \\
& \rho-\text { Air Density }\left(\mathrm{kg} / \mathrm{m}^{3}\right)
\end{aligned}
$$

The measurement uncertainty was assessed by calculating $95 \%$ confidence intervals of each coefficient using Equation 4 [24]. The maximum resulting uncertainty from these calculations for the drag coefficient is 0.001 .

$$
95 \% \text { confidence interval }= \pm 1.96 * \frac{\sigma}{\sqrt{N}}
$$

$$
\sigma-\text { Standard Deviation, } N \text { - Sample Length }
$$

\section{Pressure Measurements}

Surface static pressure measurements are achieved using a 64 channel pressure scanner via $500 \mathrm{~mm}$ long smooth bore silicone tubing. The scanner accuracy is between $\pm 0.06 \%$ and $\pm 0.1 \%$ of the full scale measurement $\left( \pm 232 \mathrm{~mm} \cdot \mathrm{H}_{2} \mathrm{O}\right)$ depending on the operating conditions. The pressure scanner was located inside the model with the communications cable exiting behind the front wheels to minimise interaction with the bulk wake flow.

The pressure scanner is a multiplexed device, so an interpolation based correction is applied to time align all 64 channels with the first channel, this is well documented by Wood [25]. Additional correction for the frequency response effects of the silicone tubing has not been applied as only mean pressure results are presented.

The locations of the surface static pressures are indicated in Figure 6, representing a $7 \times 6$ grid on the base. The pressure coefficient is calculated for each pressure tapping using Equation 5 where the total Page 3 of 7 and static measurements are taken from a pitot-static located upstream. The pressure coefficient is then corrected for blockage using the continuity correction in Equation 6.

$$
C_{p}=\frac{p-p_{s}}{\frac{1}{2} \rho v^{2}}=\frac{p-p_{s}}{p_{t}-p_{s}}
$$

$p$-is the measured pressure at the surface ( $\mathrm{Pa}$ ), subscripts of $t$ and $s$ represent the total and static measurements at the upstream location.

$$
C_{p \text { corrected }}=\frac{C_{p \text { measured }}+2 \cdot E}{1+2 \cdot E} \text { where } E=\frac{A_{m}}{A_{t}}
$$

The corrected pressure coefficients are then integrated over the base of the geometry, using Equation 7, to generate the contribution of the base pressure to the total drag of the model, this integrated value is referred to as the base drag $\left(C_{d b}\right)$. Using the analysis in Equation 4, the $95 \%$ confidence interval for the base drag is \pm 0.001 for this work.

$$
C_{d b}=\frac{1}{A_{m}} \int_{A} C_{p} \cdot d A
$$

Rather than relying on a simple geometric $0^{\circ}$ yaw condition the model is installed and the aerodynamic $0^{\circ}$ yaw condition is determined for each configuration separately by yawing the model, in small increments until a symmetric base pressure distribution is achieved. This is necessary as simplified squareback geometries are sensitive near the $0^{\circ}$ yaw condition with $0.1^{\circ}$ yaw being sufficient to force the wake into an asymmetric state $[5,26]$. This is evaluated objectively by calculating the centre of pressure on the base, using Equation 8.

$$
\begin{gathered}
\qquad O P_{y}=\frac{\sum_{i=1}^{n} \overline{C_{p i}} \cdot y_{i}}{\sum_{i=1}^{n} \overline{C_{p i}}} \\
\overline{\mathrm{C}_{\mathrm{pi}}}-\text { mean of the pressure coefficient for the } i^{\text {th }} \text { pressure tapping, } \\
\mathrm{y}_{\mathrm{i}}-\text { lateral position of the } i^{\text {th }} \text { pressure tapping }(\mathrm{m})
\end{gathered}
$$




\section{Flow Field Measurements}

The near wake flow field is measured using Particle Image Velocimetry (PIV), a non-intrusive laser based measurement method that allows the velocity field to be measured over relatively large planes, making it possible to capture snapshots of the complete wake. The planar PIV plane reported in this paper is located on the vertical centreline at $y=0 h$, from the base of the geometry to $700 \mathrm{~mm}$ downstream of the base. Two 5 megapixel sCMOS cameras as presented in Figure 7 are employed in tandem to produce a large field of view. The light sheet was created using a $525 \mathrm{~nm}$ Nd-YAG double pulsed $200 \mathrm{~mJ}$ laser passed through a plano-convex lens to generate the sheet. The sheet was approximately $1 \mathrm{~mm}$ thick in the field of view. To image the flow field, the flow was seeded with $1 \mu \mathrm{m}$ heavy alcohol (DEHS) particles using a rake located in the settling chamber and through an underfloor mounted plenum at the start of the working section. Particles were seeded throughout the test as the open return nature of the tunnel used results in negligible recycled particles.

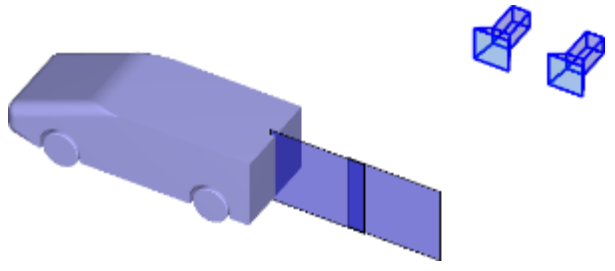

Figure 7. PIV plane location and camera set up in this work $(y=0 h)$.

During setup the inter-frame time, $(d t)$ was optimised to minimise peak locking (bias to integer pixel movements of the particles) which has a negative effect on the resulting vector field [27]. The selected time of $30 \mu \mathrm{s}$ was used throughout the results presented here. For each configuration 1000 image pairs were taken at a sampling frequency of $5 \mathrm{~Hz}$. Previous work [28] has shown 1000 image pairs to be sufficient to generate vectors that are within $\pm 2 \%$ of their true value at a $99 \%$ confidence interval.

All the images have a mean background subtraction using all images prior to processing to reduce the influence of reflections and reduce noise. The calculation of the vectors employs a multi-pass scheme for cross-correlation starting with a $256 \times 256$ pixel window with a $50 \%$ overlap reducing to a $48 \times 48$ pixel window with a $75 \%$ overlap. A q-ratio, the ratio between the highest and second highest correlation, of 1.3 was used to improve the quality of the vectors, with vectors not meeting the criteria being deleted. This processing resulted in a spatial resolution of approximately a vector every $2 \mathrm{~mm}$.

\section{Results and Discussion}

The change in drag and base drag from implementing the full span and reduced span tapering is presented in Figure 8 and Figure 9 for all configurations tested. For all taper angles, the full body tapering results in a drag reduction, as with the previous work [17], with an maximum total drag reduction of 0.018 between a taper angle of $12^{\circ}$ and $16^{\circ}$. The total drag reduction is attributed to an overall increase in base pressure, that is offset by increases in taper drag. The maximum base drag reduction of 0.039 occurs for the $20^{\circ}$ taper, but this is offset by a large taper or device drag in that case. The result is consistent with results in the literature [4-6], where larger taper angles are shown to generate more device drag providing the flow remains attached over the taper.

Page 4 of 7

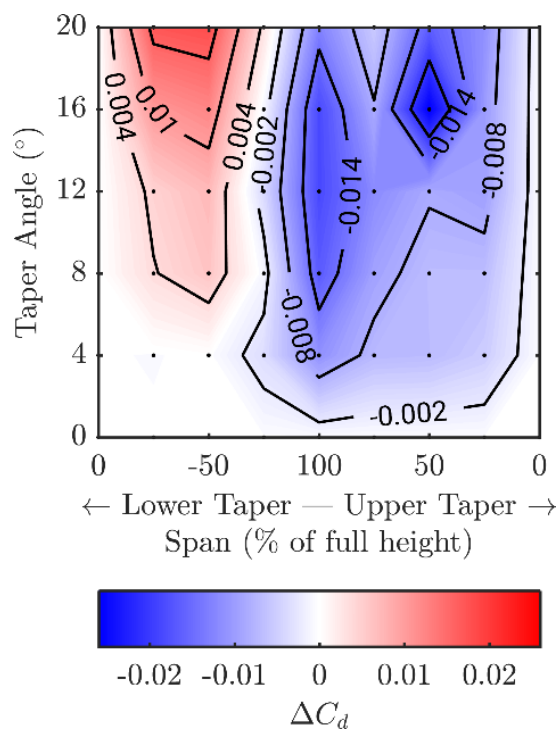

Figure 8. Change in drag coefficient with taper span and taper angle.

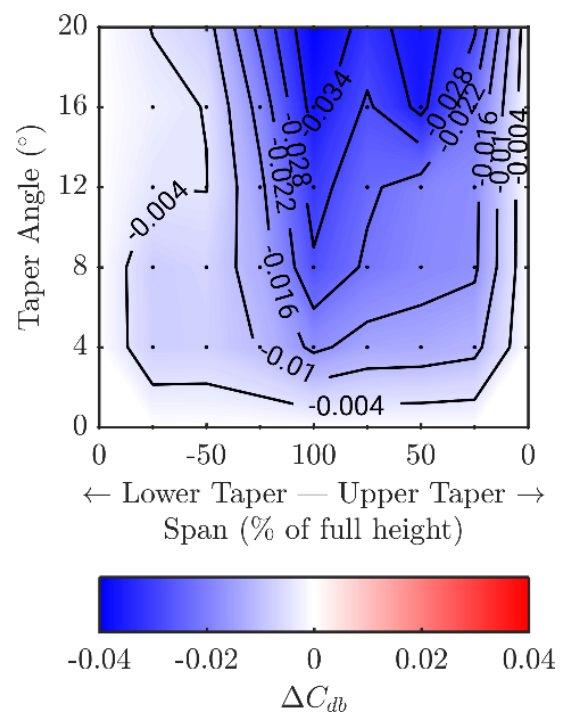

Figure 9. Change in base drag coefficient with taper span and taper angle.

When applying a partial span taper to the lower portion of the model, the maximum drag reduction is 0.003 and occurs for $\theta=4^{\circ}$ with a span of $75 \%$ of the height. This reduction is small in comparison to the drag reduction for a full height taper at the same angle where a reduction of 0.010 is achieved. In fact, for many configurations of lower taper a drag penalty occurs, whereas, the upper body tapering always produces a drag reduction, regardless of the angle or span. The overall best total drag reduction occurs for a $50 \%$ upper taper with an angle of $\theta=16^{\circ}$. This configuration generates a total drag reduction of 0.026 , considerably more than the best full span taper that gave a drag reduction of 0.018 . The $50 \%, 20^{\circ}$ upper body taper also results in a larger drag reduction than its full span equivalent, yielding a total drag reduction of 0.016 compared to 0.013 . These results differ slightly from those reported in previous work [17] , and is attributed to the to the different setup process employed here, where the symmetric base pressure distribution was found for each configuration as opposed to using a global $0^{\circ}$ yaw condition.

Considering the integrated base pressure, or base drag, Figure 9, shows that all configurations produce a reduction, ranging between $0.002\left(\theta=16^{\circ}, 25 \%\right.$ span lower tapering), to 0.039 for the full span $20^{\circ}$ taper. The upper tapers generally result in larger base drag 
reductions. The base pressure distributions (Figure 10) show that the drag reduction achieved by the $100 \%$ span tapering is a result of increasing the overall base pressure. As a reduced span lower taper is introduced, the strength of the upwash is increased affecting the strength of the impingement. With the $75 \%$ lower body taper this results in a lower pressure in the impingement region. As the span is reduced to a $25 \%$ lower body taper, the region of lowest pressure is higher up the base signifying a larger lower recirculation. On the $25 \%$ lower body taper, there is also a region of increased suction on the base of the $16^{\circ}$ and $20^{\circ}$ taper geometries close to the junction between the $0^{\circ}$ upper portion and the tapered surface. This is the effect of the longitudinal vortex described by Howell et al. [16] but it is not visible on the base pressure distributions at other span lengths. This is assumed to be a result of constructive interference with a longitudinal vortex emitted from the wheelhouse as the low pressure does not occur with other reduced spans.

When the upper body tapering is introduced, the longitudinal vortex balances the wake into a state where the upper and lower vortex structures are approximately equal in size (vertically symmetric). A vertically symmetric wake is present for a $20^{\circ}$ taper with the $25 \%$ upper span, but the $20^{\circ}$ and $16^{\circ}$ with an upper $50 \%$ taper presenting a downwash dominated wake, based on the base pressure distributions. The use of the upper body tapering presents a point that generates a switch between an upwash and downwash dominated base pressure distribution in the same way as previous work [6]. This is clear with the upwash dominated base pressure distribution on a $16^{\circ}$ taper on the upper $25 \%$ of the body. This shows that there is a compromise between the taper angle and span length in order to switch the wake position.

The total drag reduction is dependent on the balance between the improvements to the base drag and the drag introduced by the taper. This is shown when reducing the $16^{\circ}$ taper span from $100 \%$ to a $50 \%$ upper, which increased the base drag but resulted in a larger total drag reduction. It is, therefore, likely that a similar drag reduction could be achieved for several different taper angles by optimising the span to maximise the total drag reduction.

Flow field measurements on the streamwise centreline $(y=0 h)$ were performed using PIV for a selection of the $16^{\circ}$ side edge taper configurations. The flow fields are presented in Figure 11 and for comparison the base configuration without tapers is included. Tapering the upper body tends to increase the downwash in the wake, for the $25 \%$ upper taper the wake is still upwash dominated, but an increase to $50 \%$ produces a downwash dominated case. Increasing the upper taper further, to $75 \%$, returns the wake to upwash dominated. This demonstrates how the wake balance, that is closely related to the base drag, is a subtle balance between the geometry of the upper and lower body, the ground and the wheels.

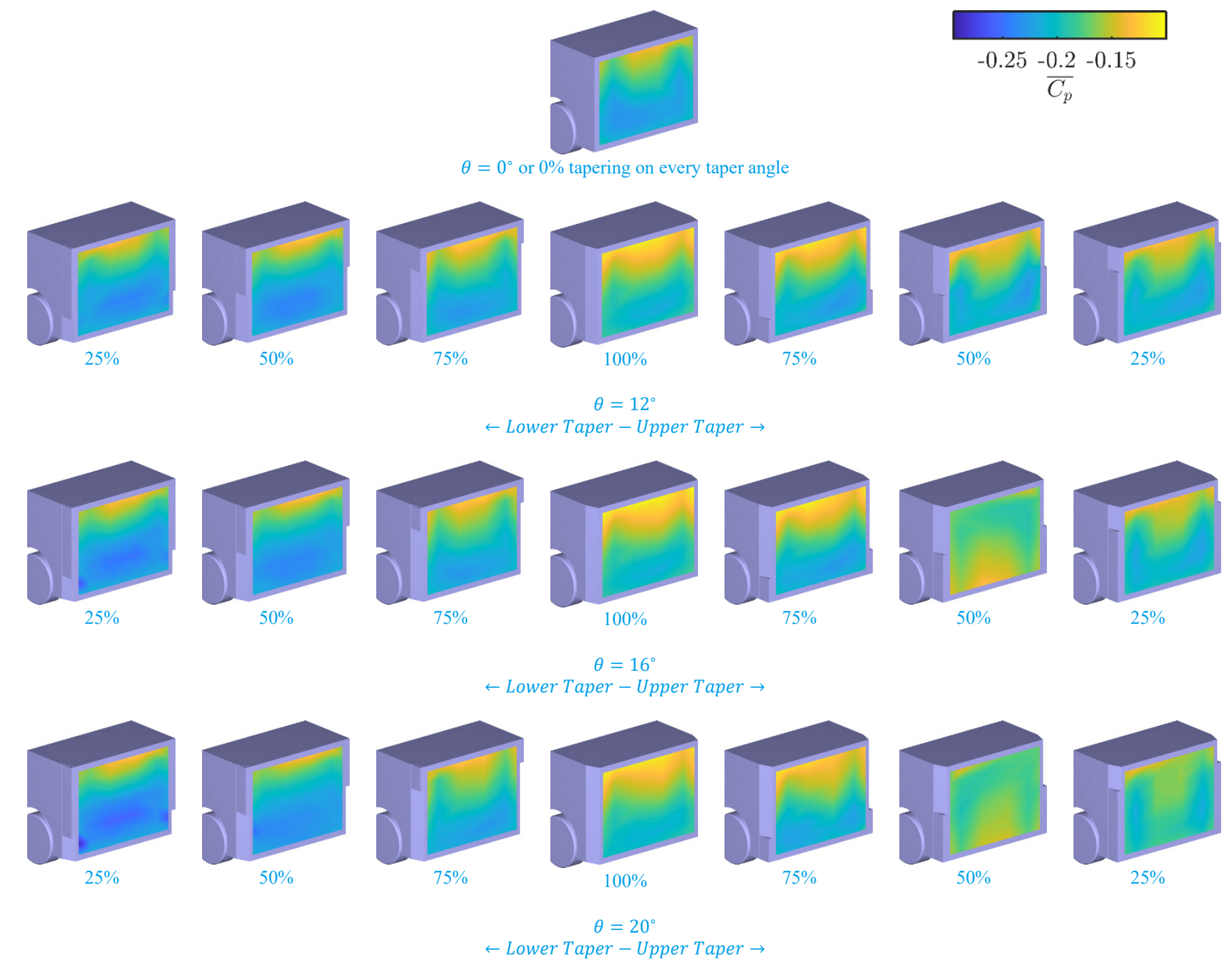

Figure 10. Base pressure distributions for the full range of taper spans for the $12^{\circ}, 16^{\circ}$ and $20^{\circ}$ taper angles.

Page 5 of 7 

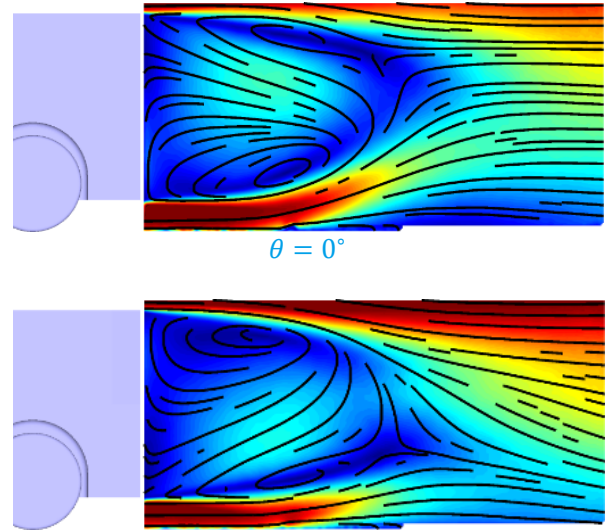

$\theta=16^{\circ}, 50 \%$ upperbody tapering

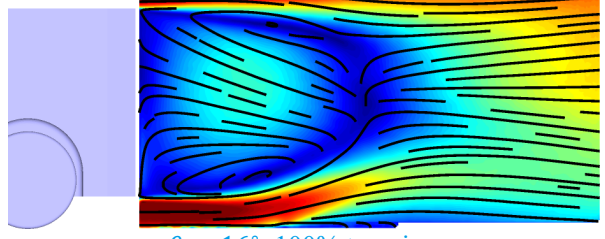

$\theta=16^{\circ}, 100 \%$ tapering

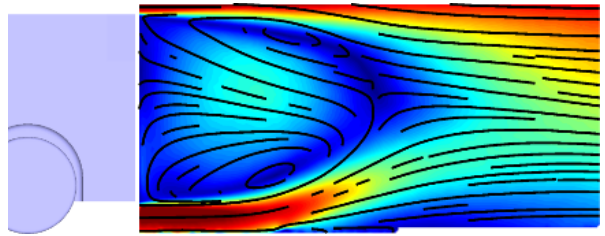

$\theta=16^{\circ}, 25 \%$ upperbody tapering

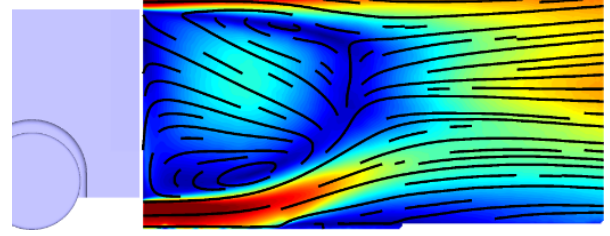

$\theta=16^{\circ}, 75 \%$ upperbody tapering



$\theta=16^{\circ}, 50 \%$ lower body tapering

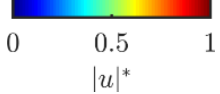

Figure 11. Normalised velocity magnitude for the $0^{\circ}$ taper and a selection of reduced span tapers based on a $16^{\circ}$ taper angle.
Given the effect of the tapers on the wake balance they also have a significant impact on the lift coefficient, presented in Figure 12. As the full span tapering is introduced on the model, the lift tends to be reduced compared to the base configuration. The reduced span tapering shows the ability to further modify the lift consistent with the changes in the upwash and downwash as the geometries with the most downwash $\left(\theta=16^{\circ}, 50 \%\right.$ upper taper $)$ and most upwash $\left(\theta=16^{\circ}\right.$, $50 \%$ lower taper) present large increases and decreases in lift respectively. There is also a small component of the lift associated with the area at the junction between the tapered and untapered surface, but as the pressures in this area are not measured, its contribution is not measured.

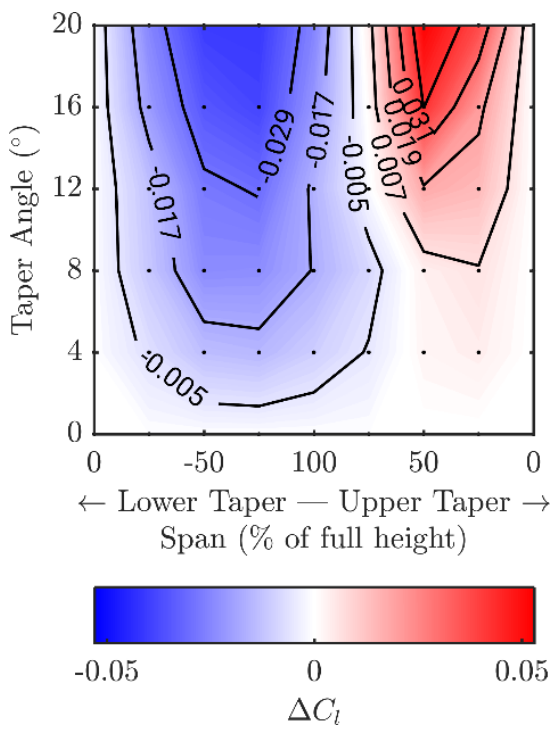

Figure 12. Change in lift coefficient with taper span and taper angle.

\section{Summary/Conclusions}

The work presented here uses force, pressures and PIV measurements to investigate the impact of applying full span and partial tapering to a simplified $1 / 4$ scale automotive geometry equipped with wheels.
- The full span taper optimum for total drag reduction is between $12^{\circ}$ and $16^{\circ}$, with a total drag reduction of 0.018 . The improvement in total drag is attributed to an increase in pressure on the base of the geometry.

- The optimum taper angle for base drag reduction is $20^{\circ}$, but the base drag reduction is offset by an increase in device drag that results in a total drag reduction of 0.039 .

- A reduced span taper can reduce the drag by more than a full span taper ( 0.026 total reduction) because it produces a more vertically balanced wake while incurring a smaller device drag penalty.

- Subtle geometric changes, in the form of reduced span tapering, have been shown to alter the wake balance and thereby reduce the base drag. This is an important consideration for the development of real vehicles, and a general result.

- The lift increase is correlated with a lower drag condition as downwash is introduced. This may have a negative impact on high speed stability if implemented on a vehicle.

- These results show the potential to allow more design freedom for full scale vehicles whilst maintaining a low drag condition.

\section{Further Work}

Any further work on these geometries should consider a smaller range of taper angles with the upper body tapering at yaw. This has shown potential in previous work and smaller increments of the reduced span may prove beneficial when relating to real vehicles.

It is unlikely that a real vehicle has no tapering on a portion of the geometry. A study that would investigate a combination of taper angles, such as a $16^{\circ}$ taper on the upper $50 \%$ of the geometry and a $12^{\circ}$ taper on the lower $50 \%$ of the geometry, may show the benefits of generating the longitudinal vortices but without the drawbacks of not tapering part of the geometry.

\section{References}

1. JATO, "Demand for SUVs slows to lowest recorded rate in June, indicating that registrations may have peaked in the European market," https://www.jato.com/demand-for-suvsslows-to-lowest-recorded-rate-in-june-indicating-that- 
registrations-may-have-peaked-in-the-european-market/, 2019.

2. Brown, Y.A.I., Windsor, S., and Gaylard, A.P., "The Effect of Base Bleed and Rear Cavities on the Drag of an SUV," SAE Technical Paper 2010-01-0512, SAE International, 2010, doi:10.4271/2010-01-0512.

3. Depardon, S., Lasserre, J., Brizzi, L.E., and Borée, J., "1/1/4 Scale Vehicle Wake Pattern Analysis using Near-Wall PIV," $S A E$ World Congr. 2006(724), 2006.

4. Pavia, G., Passmore, M., and Gaylard, A., "Influence of Short Rear End Tapers on the Unsteady Base Pressure of a Simplified Ground Vehicle," SAE Technical Paper 2016-011590, SAE International, 2016, doi:10.4271/2016-01-1590.

5. Perry, A.-K., Passmore, M., and Finney, A., "Influence of Short Rear End tapers on the Base Pressure of a Simplified Vehicle.," SAE Int. J. Passeng. Cars - Mech. Syst. 8:317-327, 2015, doi:10.4271/2015-01-1560.

6. Varney, M., Passmore, M., and Gaylard, A., "The Effect of Passive Base Ventilation on the Aerodynamic Drag of a Generic SUV Vehicle," SAE Int. J. Passeng. Cars - Mech. Syst. 10:345-357, 2017, doi:10.4271/2017-01-1548.

7. Howell, J. and Le Good, G., "The Effect of Backlight Aspect Ratio on Vortex and Base Drag for a Simple Car-Like Shape," SAE Technical Paper 2008-01-0737, SAE International, 2008, doi:10.4271/2008-01-0737.

8. Grandemange, M., Mary, A., Gohlke, M., and Cadot, O., "Effect on drag of the flow orientation at the base separation of a simplified blunt road vehicle," Exp. Fluids 54(5), 2013, doi:10.1007/s00348-013-1529-z.

9. Ahmed, S.R., Ramm, G., and Faltin, G., "Some Salient Features Of The Time-Averaged Ground Vehicle Wake," SAE Technical Paper 840300, SAE International, 1984, doi:10.4271/840300.

10. Littlewood, R. and Passmore, M., "The Optimization of Roof Trailing Edge Geometry of a Simple Square-Back.," $S A E$ Technical Paper 2010-01-0510, SAE International, 2010, doi:10.4271/2010-01-0510.

11. Evrard, A., Cadot, O., Herbert, V., Ricot, D., Vigneron, R., and Délery, J., "Fluid force and symmetry breaking modes of a 3D bluff body with a base cavity," J. Fluids Struct. 61:99-114, 2016, doi:10.1016/j.jfluidstructs.2015.12.001.

12. Howell, J., Sims-Williams, D., Sprot, A., Hamlin, F., and Dominy, R., "Bluff Body Drag Reduction with Ventilated Base Cavities," SAE Int. J. Passeng. Cars - Mech. Syst. 2012-010171 5:152-160, 2012, doi:10.4271/2012-01-0171.

13. Duell, E.G. and George, A.R., "Experimental Study of a Ground Vehicle Body Unsteady Near Wake," SAE Technical Paper, SAE International, 1999, doi:10.4271/1999-01-0812.

14. Modi, V.J., "Moving Surface Boundary-Layer Control : a Review,” J. Fluids Struct. 11:627-663, 1997, doi:10.1006/jfls.1997.0098.

15. Seifert, A., Shtendel, T., and Dolgopyat, D., "From lab to full scale Active Flow Control drag reduction: How to bridge the gap?," J. Wind Eng. Ind. Aerodyn. 147:262-272, 2015, doi:https://doi.org/10.1016/j.jweia.2015.09.012.

16. Howell, J., Passmore, M., and Tuplin, S., "Aerodynamic Drag Reduction on a Simple Car-Like Shape with Rear Upper Body Taper," SAE Int. J. Passeng. Cars - Mech. Syst. 6:52-60, 2013, doi:10.4271/2013-01-0462.

17. Varney, M., Passmore, M., and Gaylard, A., "Parametric Study

Page 7 of 7 of Asymmetric Side Tapering in Constant Cross Wind Conditions," WCX World Congr. Exp. 11(3):213-223, 2018, doi:https://doi.org/10.4271/2018-01-0718.

18. Pavia, G., Passmore, M., and Varney, M., "Low-frequency wake dynamics for a square-back vehicle with side trailing edge tapers," J. Wind Eng. Ind. Aerodyn. 184(December 2018):417-435, 2019.

19. Li, R., Borée, J., Noack, B.R., Cordier, L., and Harambat, F., "Drag reduction of a yawed car model by combining fluidic flaps and turbulence control," ISBN 9788894364200: Presentation Only, 2018.

20. Garcia de la Cruz, J.M., Brackston, R.D., and Morrison, J.F., "Adaptive Base-Flaps Under Variable Cross-Wind," SAE Tech. Pap. Ser. 1, 2017, doi:10.4271/2017-01-7000.

21. Johl, G., "The Design and Performance of a $1.9 \mathrm{mx} 1.3 \mathrm{~m}$ Indraft Wind Tunnel," phdthesis, Ph.D. thesis, Aeronautical and Automotive Engineering, Loughborough University, 2010.

22. SAE International Vehicle Aerodynamics Terminology, SAE Standard J1594, Rev Dec. 1994.

23. Carr, G.W. and Stapleford, W.R., "Blockage Effects in Automotive Wind-Tunnel Testing," SAE International Congress and Exposition, SAE International, 1986, doi:10.4271/860093.

24. Coleman, H.W. and Steele, W.G., "Experimentation, validation, and uncertainty analysis for engineers," John Wiley \& Sons, 2009.

25. Wood, D., "The Effect of Rear Geometry Changes on the Notchback Flow Field," phdthesis, Loughborough University, 2015.

26. Pavia, G., Passmore, M., and Sardu, C., "Evolution of the bistable wake of a square-back automotive shape," Exp. Fluids 59(1):1-20, 2018, doi:10.1007/s00348-017-2473-0.

27. Adrian, L., Adrian, R.J., and Westerweel, J., "Particle Image Velocimetry," Cambridge University Press, ISBN $9780521440080,2011$.

28. Hollis, D., "Particle image velocimetry in gas turbine combustor flow fields," phdthesis, Loughborough University, 2004.

\section{Contact Information}

Max Varney, Stewart Miller Building, Loughborough University, Loughborough, LE11 3TU, England. M.Varney@lboro.ac.uk

Martin Passmore, M.A.Passmore@1boro.ac.uk

\section{Acknowledgements}

This work was supported by Jaguar Land Rover and the UK EPSRC under an industrial CASE aware (Voucher No. 15220097). Thanks also go to Andrew Horsey for manufacturing the parts and providing mechanical support. 